Canadian Oncology

Nursing Journal

Revue canadienne

de soins infirmiers

en oncologie

Volume 27, Issue 3 • Summer 2017

elSSN: $2368-8076$ 


\section{An implementation evaluation of a wellness beyond cancer program survivorship class: Who is attending?}

by Georden Jones, Caroline Séguin Leclair, Danielle Petricone-Westwood, Monique Lefebvre, Robin Morash, Carrie Liska, Lynne Jolicoeur, and Sophie Lebel

\begin{abstract}
Breast and endometrial cancer survivors referred to the Wellness Beyond Cancer Program were invited to a survivorship education class. As not all survivors attended the class, this study aimed to identify socio-demographic and medical characteristics, and survivorship needs that predict attendance. A chart review was conducted on survivors who completed a needs assessment survey between 2012 and 2014 ( $n=144$ endometrial; $n=170$ breast). Class attendees' characteristics were compared to those of non-attendees using t-tests, chi-square analyses, and regression analysis.
\end{abstract}

\section{ABOUT THE AUTHORS}

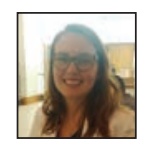

Georden Jones*, BA, School of Psychology, University of Ottawa, 136 Jean Jacques Lussier PVT, Room 4016, Ottawa, ON K1N 6N5

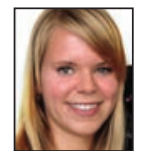

Caroline Séguin Leclair, BSc, School of Psychology, University of Ottawa, 136 Jean Jacques Lussier PVT, Room 4016, Ottawa, ON K1N 6N5

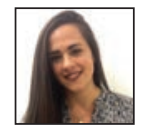

Danielle Petricone-Westwood, BA, School of Psychology, University of Ottawa, 136 Jean Jacques Lussier PVT, Room 4016, Ottawa, ON K1N 6N5

Monique Lefebvre, PhD, CPsych, The Ottawa Hospital Cancer Centre, The Ottawa Hospital, Ottawa, ON

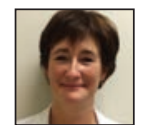

Robin Morash, RN, BNSc, MHS, The Ottawa Hospital Cancer Centre, The Ottawa Hospital, Ottawa, ON

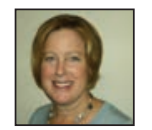

Carrie Liska, RN, BScN, MN, The Ottawa Hospital Cancer Centre, The Ottawa Hospital, Ottawa, ON

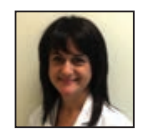

Lynne Jolicoeur, RN, MScN, CON(C), The Ottawa Hospital Cancer Centre, The Ottawa Hospital, Ottawa, ON

Sophie Lebel, PhD, CPsych, School of Psychology, University of Ottawa, 136 Jean Jacques Lussier PVT, Room 4016, Ottawa ON K1N 6N5

Correspondence: Ms. Georden Jones, B.A., School of Psychology, University of Ottawa, 136 Jean Jacques Lussier PVT, Room 4016, Ottawa ON K1N 6N5. Ph: 613-562-5800 ext. 7152

For questions related to the Wellness Beyond Cancer Program, please contact Robin Morash: rmorash@toh.ca

DOI: $10.5737 / 23688076273268274$
Univariate analyses showed that age, distance from hospital, emotional and physical needs, and receiving chemotherapy and/or radiation therapy were associated with class attendance. Distance from hospital and physical needs were identified as multivariate predictors. The results of this study will help inform class content, improve class attendance, and identify attendees' characteristics.

Key words: survivorship; care transitions; follow-up care; breast cancer; endometrial cancer

7 The Canadian Cancer Society (CCS) estimates that 25,000 1 women were diagnosed with breast cancer in 2015 and that $88 \%$ will survive five years post diagnosis (CCS, 2015a). It is estimated that 1 in 36 Canadian women will be diagnosed with uterine cancer, which includes endometrial cancer, during their lifetime (CCS, 2015b). A large number of breast and endometrial cancer survivors will therefore require follow-up care.

\section{Survivorship Follow-Up Care}

Given the increasing number of cancer survivors, who are defined as patients who were diagnosed with cancer and have now completed their primary cancer treatments (Feuerstein, 2007), and given their increase in survival rates, the demand for follow-up care is increasing. The main goals of follow-up care are symptom surveillance to facilitate early detection of recurrence, management of treatment side effects, and education. Cancer survivors report experiencing many outstanding needs for up to 10 years post diagnosis, in particular access to improved team care and communication between healthcare providers (Hodgkinson, Butow, Fuchs, Hunt, Stenlake, Hobbs, et al., 2007). It is, thus, important to prepare and facilitate survivors' transition from tertiary care to follow-up in primary care settings.

\section{Cancer Survivors' Unique Needs}

Several studies have established that breast cancer survivors have many outstanding needs (Armes et al., 2009) and face various challenges in survivorship surrounding their physical health, finances, future plans, and family (Lebel, Rosberger, Edgar, \& Devins, 2007; Stalker, Johnson, \& Cimma, 1990). Similarly, endometrial cancer survivors reported having emotional, physical, medical, and informational needs post-treatment (Hodgkinson et al., 2007). Addressing cancer survivors' outstanding needs is, therefore, of great importance in follow-up care (Cox et al., 2006; Grunfeld et al., 2006; Kelly, Caldwell, \& Henshaw, 2006). Some of the most common informational needs identified for gynecological cancer survivors include general medical information about cancer, 
the likelihood of the development of a cure, the management of symptom side effects, the prevention of a recurrence, and coping with fear of cancer recurrence (Papadakos et al., 2012). Breast cancer survivors have also reported similar informational needs (Fiszer, Dolbeault, Sultan, \& Brédart, 2014; Kelly et al., 2006; Tariman, Doorenbos, Schepp, Singhal, \& Berry, 2014), as well as psychological needs and wanting information on the healthcare system (Fiszer et al., 2014). Unfortunately, literature has shown that cancer survivors often feel unsatisfied with the information provided in follow-up care and wish to have more educational content (Armes et al., 2009; Kelly et al., 2006; Mallinger, Griggs, \& Shields, 2005; Nicolaije et al., 2012; Papadakos et al., 2012).

\section{Survivorship Models of Care}

Many hospitals have begun implementing programs to address cancer survivors' post-treatment needs. Although the numbers of survivorship programs are increasing, literature on the topic is sparse. Two program evaluation reports in particular have been published, both describing and evaluating supportive oncology programs for cancer patients at Stanford University (see Risendal et al., 2014, and Rosenbaum et al., 2004, for full descriptions and results).

The objective of the first program, the Stanford Cancer Supportive Care Program, was to support cancer patients throughout treatment and post-treatment by addressing quality-of-life needs (Rosenbaum et al., 2004). The majority of attendees were older than 51 years of age, female, and primarily diagnosed with breast cancer. Caucasian and Asian patients were found to have participated in the program more often than other ethnic groups. Researchers evaluated the feasibility and acceptability of the second program, the Cancer Thriving and Surviving Program (Risendal et al., 2014), which had the objective of promoting successful coping and recovering well-being. Attendees were mostly between the ages of 50 and 64 years old, Caucasian, and married. The class attendees had various cancer diagnoses, although most had breast cancer and were one to three years post-diagnosis. Both these programs reported high levels of satisfaction from participants, as they target quality of life in terms of physical and emotional functioning. However, physical and emotional needs were not measured in the evaluation. To ensure that patients transition smoothly to primary care, program developers must ensure that survivorship care needs are being addressed.

\section{The Wellness Beyond Cancer Program}

The Wellness Beyond Cancer Program (WBCP) is a cancer survivorship program at The Ottawa Hospital Cancer Centre (TOHCC) that consists of a multidisciplinary team of healthcare providers. Currently the WBCP provides survivorship care to colorectal, breast, and endometrial cancer patients. The purpose of the program is to ensure, at the end of active treatment, that patients have access to resources that best meet their individual needs. Patients are referred to the WBCP by their oncologist and are asked to complete a needs assessment at the time of referral. The needs assessment and referral form are sent to the WBCP. Outstanding self-identified needs indicated by the patient as being moderate to extreme are captured and included in the patients' individualized care plan by an oncology nurse. The breast disease site program model varies slightly from the endometrial model.

Breast cancer patients are referred by their oncologist to follow-up survivorship care provided by (a) their Primary Care Provider (PCP), or (b) a WBCP Nurse Practitioner. Selection of either of these discharge streams is based on a clinical assessment of risk of recurrence and/or complexity of the patient being referred (Rushton et al., 2015). Regardless of the stream, the goal is to transition care back to the PCP. Breast cancer patients are invited to attend an education class and are subsequently scheduled for a discharge visit. At the discharge visit, an oncology nurse reviews their individualized survivorship care plan and symptom management strategies are reviewed to address any self-identified outstanding physical or psychosocial needs. The survivorship care plan is provided to the patient to take home following the visit. A copy of the care plan is then mailed to the PCP.

Endometrial cancer patients are referred to a single stream for follow-up survivorship care provided by the PCP. Endometrial patients are invited to attend an education class. The individualized care plan is reviewed in a group setting at the conclusion of the education class. Patients are offered the opportunity to address any outstanding self-identified physical or psychosocial needs privately following the education class. Survivors are provided contact numbers of the nurses for any follow-up questions, and are encouraged to schedule their follow-up appointments with their PCP. For those patients who do not attend the class, their care plan is mailed to both the patient and their PCP.

\section{The Wellness Beyond Cancer Program Survivorship Class}

Disease-specific classes are implemented to facilitate the communication of relevant cancer survivorship information, and to foster empowerment and well-being (see Rushton et al., 2015). The WBCP education class is a one-time 90 to 120 -minute session offered by an oncology nurse. The class topics include the coordination of follow-up care, signs and symptoms of recurrence for monitoring purposes, recommendations for other cancer screening, healthy lifestyle recommendations, and the most common physical and psychosocial late and long-terms effects due to cancer treatment. Examples of late and long-term effects include fear of recurrence, body image and sexuality issues, fatigue, peripheral neuropathy and menopausal symptoms. The process for PCPs to call with questions or concerns, and re-entry to the cancer program if necessary, is also detailed. The time from end of treatment to referral to WBCP varies depending on the complexity of treatments received, patients' treatment sequelae, and individual oncologists' preferences. Endometrial cancer classes are offered at a local cancer survivorship community centre between 3:00 p.m. and 5:00 p.m. once every two months. Due to larger patient numbers, breast cancer classes are scheduled more frequently: monthly from 1:00 p.m. to 3:00 p.m. at the cancer centre, at either an east- or west-end location of the city. 


\section{Study Objectives}

The purpose of this study was to determine characteristics of breast and endometrial patients associated with attendance at the Wellness Beyond Cancer Program (WBCP) survivorship class. In particular, we sought to identify socio-demographic characteristics, medical characteristics and cancer survivors' needs associated with survivors' attendance. We hypothesized that younger patients, those living geographically closer to the hospital, and those reporting greater survivorship needs would be more likely to attend a class, as recommended by their team. The influence of other variables on class attendance, such as marital status, ethnicity, having children, cancer diagnosis, type of treatment and year since cancer diagnosis was also explored.

\section{METHODS}

\section{Procedure}

A retrospective data analysis was conducted to determine which socio-demographic characteristics, medical characteristics and self-reported outstanding needs were associated with attendance to the WBCP survivorship class. Ethics approval was obtained from the Ottawa General Hospital Research and Ethics Board. The following inclusion criteria were used to select patients: diagnosed with breast or endometrial cancer, discharged from the TOHCC, and referred by oncologists to the WBCP survivorship class between 2012 and 2014. The WBCP patient referral database at the TOHCC was used to select the participants. For the breast cancer group, a list of 250 patients was obtained; those missing medical data (i.e., treatment received, diagnosis) were removed leaving a sample of 170 breast patients. For the endometrial patients, a list of 170 patients was obtained. Twenty-six patient files had insufficient medical data, which reduced the endometrial cancer sample to 144 patients. Chart reviews were performed to collect the data from electronic charts. The extracted data were entered into an IBM SPSS v24 database for analyses.

\section{Measures}

Socio-demographics and medical characteristics. Medical charts were consulted to extract the following socio-demographic and medical variables: date of birth, postal code, type of cancer, year of diagnosis, and type of treatment received. The remaining socio-demographic variables (ethnicity, marital status and having children) were obtained from the demographics section of the individual Needs Assessment Survey (see below) completed by participants after discharge from the TOHCC.

Needs assessment survey. The needs assessment survey (Mayo Clinic, N.D.) is a validated assessment tool that asks patients to indicate their levels of concern in a number of areas grouped into five subscales (emotional, physical, spiritual, social and other). The patients report their outstanding symptom concerns on a Likert scale from 0 (no concerns) to 5 (extreme concerns). This questionnaire is administered as part of usual care at the last appointment at the cancer centre. The needs assessment is completed by the patient at the time of referral to the WBCP and the needs assessment and program referral are subsequently sent to the WBCP office.
Analyses. Analyses were conducted using IBM SPSS v24 following data extraction. T-test analyses were used to compare attenders to non-attenders on various continuous variables, and chi-square tests were used to compare on dichotomous variables. A multiple hierarchical logistic regression was performed using significant variables from the first analyses, to identify any multivariate predictors of attendance to the class. The sample size $(n=314)$ was sufficiently large to perform the regression with six predictors, following the norms established by Tabachnick, Fidell and Osterlind (2001) (that is, $50+$ $8^{*}$ number of predictors $\left.(6)=98\right)$.

\section{RESULTS}

\section{Data Cleaning}

There were no other missing data from the breast cancer survivors, whereas the endometrial group was missing data. Only 14 needs assessment surveys for endometrial cancer survivors were entered into the electronic medical charts, therefore data on ethnicity, marital status and having/not having children were not included in the analyses for the endometrial patients. Some of the data were transformed for the analyses: the patients' date of birth was converted into the patient's age at the time of the class, postal code was converted into distance from the patient's residence to the hospital, and ethnicity was combined into a dichotomous variable (white and other), because few participants endorsed non-white categories. One outlier was removed related to residence distance from hospital variable.

The needs assessment information for the endometrial cancer patients was recovered in nursing notes only for scores rated above 3. Therefore, each needs assessment item was dichotomized as a reported need ( 3 or more) or non-reported need (less than 3), for both the breast and endometrial cancer survivor groups. Totals for the number of reported needs were computed for each subscale (i.e., physical, emotional, social, spiritual and other).

\section{Sample}

A total of 314 cancer survivors were included in the analyses ( $\mathrm{n}=170$ breast, $\mathrm{n}=144$ endometrial). Out of the total sample, 136 patients attended the class $(43.3 \%)$. The mean age of the sample was 63 years old $(\mathrm{SD}=10.92)$ and the average time between diagnosis and class attendance was 3.44 years $(\mathrm{SD}=3.24$ ) (see Table 1 for socio-demographic and medical results).

\section{Socio-demographic Variables}

For the overall sample, patients who attended the WBCP survivorship class were significantly younger $(\mathrm{t}(312)=2.56, \mathrm{p}=.01)$ and lived closer to the hospital $(\mathrm{t}(311)=4.99, \mathrm{p}<.001)$ than non-attenders. For the breast cancer patients, ethnicity $\left(\chi^{2}(1)=0.01\right.$, $\mathrm{p}=.92)$, marital status $\left(\chi^{2}(3)=2.56, \mathrm{p}=.18\right)$, and having children $\left(\chi^{2}(1)=.60, p=.44\right)$ had no impact on patient attendance.

\section{Medical and Disease-related Variables}

For both cancer sites, having received chemotherapy $\left(\chi^{2}(1)=5.10, p=.02\right)$ and having received radiation therapy $\left(\chi^{2}(1)=6.94, p=.01\right)$ were associated with patient attendance at the class. By contrast, having undergone surgery $\left(\chi^{2}(1)=2.48\right.$, 
$\mathrm{p}=.12)$, having received hormone therapy $\left(\chi^{2}(1)=1.85, \mathrm{p}=.17\right)$, having breast cancer $\left(\chi^{2}(1)=3.66, p=.06\right)$, and time since cancer diagnosis $(\mathrm{t}(310)=-0.26, \mathrm{p}=.80)$ were not associated with patient attendance.

\section{Needs Assessment Variables}

Overall, physical $(\mathrm{t}(275)=-3.43, \mathrm{p}=.00)$ and emotional needs $(\mathrm{t}(272)=-2.19, \mathrm{p}=.03)$ were associated with attendance at the WBCP survivorship class. However, social $(\mathrm{t}(276)=-1.63, \mathrm{p}=.10)$, spiritual $(\mathrm{t}(282)=0.71, \mathrm{p}=.48)$, and other $(\mathrm{t}(142)=-1.54, \mathrm{p}=.13)$ needs were not associated with attendance. Table 2 presents the reported frequency for all items of the Needs Assessment Survey.

\section{Multiple Hierarchical Regression}

The multiple hierarchical logistical regression was run using the significant univariate predictors of class attendance. In the first block, distance from hospital and age were entered.

\section{Table 1: Descriptive Statistics}

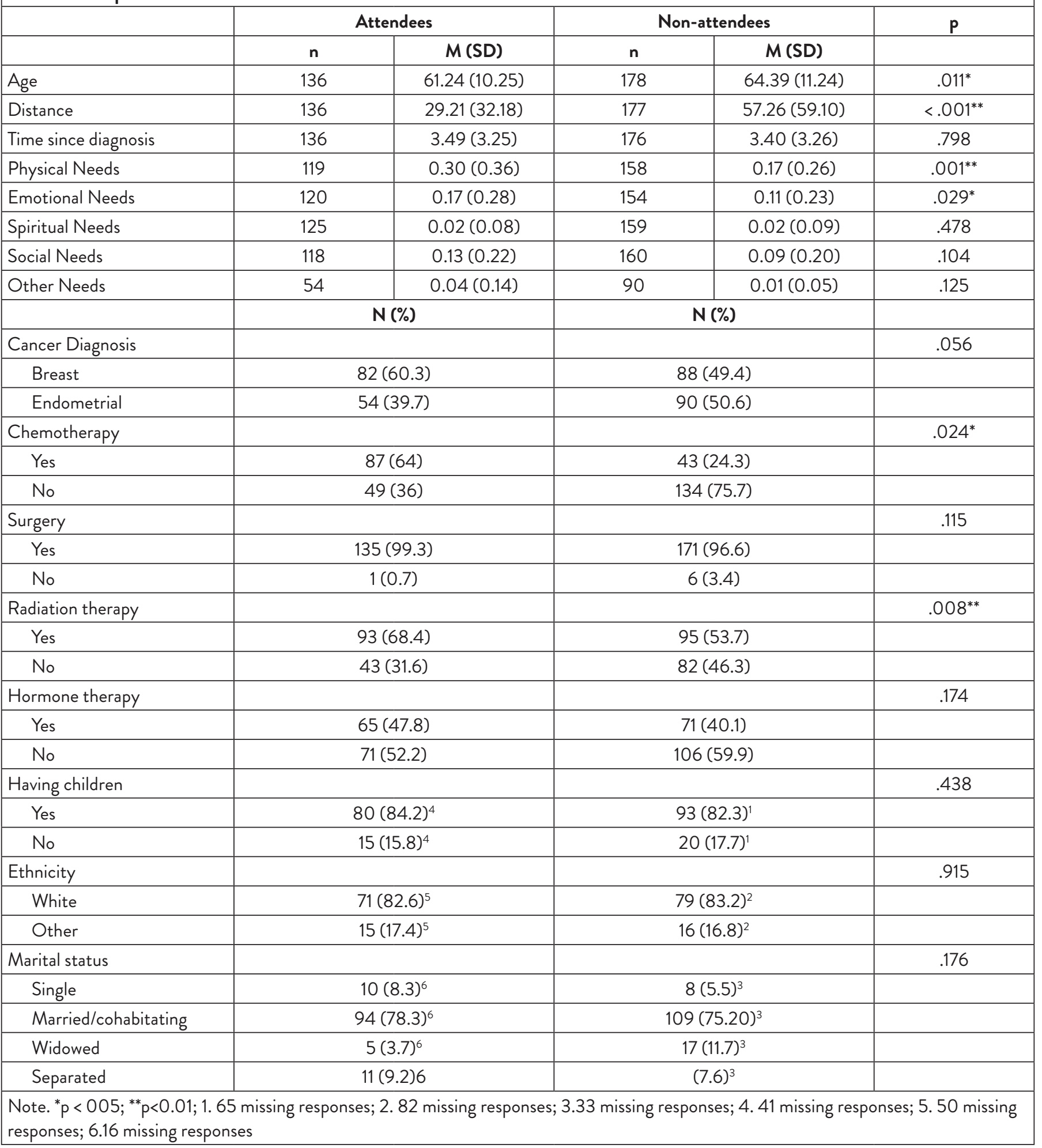




\begin{tabular}{|l|c|c|c|}
\hline \multicolumn{4}{|c|}{ Table 2: Multivariate predictors of class attendance } \\
\hline & $\boldsymbol{\beta}$ & $\mathbf{d f}$ & $\mathbf{P}$ \\
\hline Distance & -.015 & 1 & .000 \\
\hline Age & -.017 & 1 & .225 \\
\hline Chemotherapy & -.107 & 1 & .758 \\
\hline Radiation & .435 & 1 & .137 \\
\hline Physical Needs & 1.544 & 1 & .015 \\
\hline Emotional Needs & -.309 & 1 & .686 \\
\hline Constant & .855 & 1 & .355 \\
\hline
\end{tabular}

In the second block, chemotherapy and radiation therapy were entered. In the last block, physical and emotional needs were entered. Overall, this model was significant $\left(\chi^{2}(6)=37.09\right.$, $\mathrm{p}<0.001, \mathrm{R} 2=18.4 \%)$. Distance from hospital $(\beta=-0.015$, $\mathrm{p}<0.001)$ and physical needs $(\beta=1.54, \mathrm{p}=0.02)$ were significant multivariate predictors of attendance to the class. Age $(\beta=-0.017, p=0.23)$, chemotherapy $(\beta=-0.107, p=0.76)$, radiation therapy $(\beta=0.435, p=0.14)$ and emotional needs $(\beta=-0.309, p=0.69)$ were not found to be significant predictors of class attendance. Therefore, living closer to the hospital and having more physical needs increases the odds of patients attending the workshop. (See Table 3.)

Table 3: Reported frequencies of survivorship needs

\begin{tabular}{|c|c|c|c|c|c|}
\hline Need & Endorsing \% & Missing \% & Need & Endorsing \% & Missing \% \\
\hline Fatigue & 16.6 & 0.3 & Managing Stress & 11.8 & 1.3 \\
\hline Sleep & 14.6 & 3.5 & Counselling Services & 3.8 & 2.2 \\
\hline Sexual Issues & 9.2 & 2.9 & Changing Relationships & 4.8 & 2.2 \\
\hline Balance and Walking & 8.3 & 1.9 & Loss of Faith & 1.3 & 3.8 \\
\hline Bowel and Bladder & 7.6 & 1.3 & Religious Distress & 0 & 3.5 \\
\hline Weight & 10.5 & 1.6 & End of Life Distress & 2.9 & 2.9 \\
\hline Swelling & 8.6 & 1.3 & Caring for Family & 7 & 1.3 \\
\hline Hot Flashes & 15.0 & 1.9 & Fertility & 0.6 & 3.5 \\
\hline Swallowing & 0.6 & 1.3 & Genetic Counselling & 11.1 & 1.6 \\
\hline Hair and Skin & 6.1 & 2.2 & Talking about Cancer & 2.9 & 0.6 \\
\hline Dental & 3.8 & 1.6 & Returning to Work & 5.1 & 5.4 \\
\hline Osteoporosis & 9.9 & 1 & Extended Health Insurance & 4.1 & 3.5 \\
\hline Memory and Concentration & 11.8 & 0.6 & Legal Concerns & 1.3 & 1.9 \\
\hline Coping & 7 & 1.3 & Primary Care Provider Informed & 8.3 & 1.6 \\
\hline Support Resources & 3.5 & 1.6 & Complementary and Alternative Medicine & 2.9 & 3.2 \\
\hline Uncertainty & 8.9 & 1.6 & Long Term effects & 1.6 & 0.3 \\
\hline Fear of Cancer Recurrence & 17.5 & 0.6 & Sense of Wellbeing & 5.7 & 0.3 \\
\hline
\end{tabular}




\section{DISCUSSION}

This study identified those patient variables associated with attendance to the WBCP survivorship class that may help in developing similar programs and informing survivorship care. As expected, younger age and shorter living distance from the hospital were associated with class attendance. This is concordant with previous literature that found younger age to be related to higher needs (Fiszer et al., 2014; Hodgkinson et al., 2007; Lebel et al., 2013). The hypothesis that survivors who report higher needs are more likely to attend the class was supported: survivors who reported higher physical and emotional needs did tend to attend the class more than those who reported fewer needs in these domains. These results corroborate the literature, which has shown that higher physical and psychological needs are associated with more informational needs (Armes et al., 2009; Rutten, Arora, Bakos, Aziz, \& Rowland, 2005), suggesting that the class may be perceived as a way to address these outstanding informational needs. Indeed, previous literature review demonstrated that younger patients, patients with higher needs, more educated patients, and those of higher socio-economic status tend to seek information from diverse sources, such as information sessions. This may explain our finding that younger survivors and survivors with higher emotional and physical needs were more likely to attend the class. Finally, patients who received chemotherapy and/or radiation therapy were more likely to attend the WBCP class than those who did not. These results could be related to greater physical side effects among patients receiving such treatments (Canadian Cancer Society, 2016; Griffin et al., 1996).

These results suggest that survivorship programs such as the WBCP survivorship class could be tailored to younger cancer survivors, who often have different needs or areas of concern (e.g., child care, infertility, internet resources, and family counselling; Zebrack, 2009). Moreover, it is possible that older survivors had lower attendance rates due to physical mobility barriers or other chronic illnesses (SteinhagenThiessen \& Borchelt, 1999). This would indicate the need to reach out in other ways to these survivors to ensure adequate follow-up care and accessibility of services. With respect to the latter, it is recommended that programs collaborate with social services, such as transportation services, to reduce some of the barriers to accessing the class. In addition, the results show that survivors who live further away are less likely to attend the class. As the regional cancer centre has a very large catchment area, other methods of providing information should, thus, also be considered for (e.g., online modules, videoconference or satellite clinics). Progress has been made in this regard, with the WBCP survivorship classes now being offered through videoconferencing for rural patients, based on informal feedback. The implementation and outcomes of this class modality should be investigated in the future. The results of this study also suggest that time since diagnosis, type of cancer, ethnicity, marital status, having children, social and spiritual needs do not as heavily influence survivors' attendance to the class.

\section{Limitations}

The limitations of the present study include the use of a cross-sectional design, which limits any conclusion on causal relationships. The majority of attendees were breast cancer survivors, and despite the difference being non-significant $(p=.06)$, results may be more representative of breast cancer survivors than endometrial survivors. The missing data for endometrial patients' socio-demographic variables limit the conclusions drawn surrounding these characteristics. Some socio-demographic information was not available, such as mother tongue and socioeconomic status, therefore conclusions about whether these variables may help predict class attendance could not be drawn. In addition, it is possible that organizational factors such as the time and the location of the class may have influenced survivors' attendance. In particular, the late-afternoon timing of the classes may have been difficult for survivors to attend due to traffic, family obligations or fatigue. Finally, since class attendance was dependent upon referral from oncologists, factors influencing oncologists' willingness to refer patients to survivorship programs should be further investigated.

\section{Future Directions}

Qualitative interviews with patients being referred to the program may allow further understanding of class attendance and the identification of barriers to class attendance. Moreover, future research should evaluate whether the class curriculum is meeting the patients' needs by conducting follow-up data collection. This might inform us as to whether the information provided is adequate and if it meets their longer-term survivorship needs. Furthermore, investigation of the benefit of the survivorship care plans in the transition of care to the PCP should be performed. Finally, other factors that may increase satisfaction of outstanding needs, such as the provision of paper handouts, follow-up calls or different formats of the class, should be considered in future research.

\section{CONCLUSIONS}

This study aimed to identify cancer survivor characteristics associated with attendance at the WBCP survivorship class. Younger age, residing closer to the hospital, having undergone chemotherapy and/or radiation, and having higher physical and emotional needs were all significantly associated with a greater likelihood of attendance. The identification of such characteristics provides insight into the most typical class attendee. This information is an initial step in determining how best to promote the benefit of attending these cancer survivorship classes. 


\section{REFERENCES}

Armes, J., Crowe, M., Colbourne, L., Morgan, H., Murrells, T., Oakley, C., ... Richardson, A. (2009). Patients' supportive care needs beyond the end of cancer treatment: a prospective, longitudinal survey. Journal of Clinical Oncology, 27(36), 6172-6179. http://doi. org/10.1200/JCO.2009.22.5151

Canadian Cancer Society. (2015a). Breast cancer statistics. Retrieved from http://www.cancer.ca/en/cancer-information/cancer-type/ breast/statistics/?region $=\mathrm{ab}$

Canadian Cancer Society. (2015b). Uterine cancer statistics. Retrieved from http://www.cancer.ca/en/cancer-information/cancer-type/ uterine/statistics/?region=on

Canadian Cancer Society. (2016). Side effects of radiation therapy. Retrieved from http://www.cancer.ca/en/cancerinformation/diagnosis-and-treatment/radiation-therapy/ side-effects-of-radiation-therapy/?region $=$ on

Cox, K., Wilson, E., Heath, L., Collier, J., Jones, L., \& Johnston, I. (2006). Preferences for follow-up after treatment for lung cancer: Assessing the nurse-led option. Cancer Nursing, 29(3), 176-187.

Fiszer, C., Dolbeault, S., Sultan, S., \& Brédart, A. (2014). Prevalence, intensity, and predictors of the supportive care needs of women diagnosed with breast cancer: A systematic review. PsychoOncology, 23(4), 361-374. http://doi.org/10.1002/pon.3432

Feuerstein, M. (2007). Defining cancer survivorship. Journal of cancer survivorship: research and practice, 1(1), 5-7

Griffin, A.M., Butow, P.N., Coates, A.S., Childs, A.M., Ellis, P.M., Dunn, S.M., \& Tattersall, M.H.N. (1996). On the receiving end V: Patient perceptions of the side effects of cancer chemotherapy in 1993. Annals of Oncology, 7(2), 189-195.

Grunfeld, E., Levine, M.N., Julian, J.A., Coyle, D., Szechtman, B., Mirsky, D., ... Whelan, T. (2006). Randomized trial of long-term follow-up for early-stage breast cancer: A comparison of family physician versus specialist care. Journal of Clinical Oncology, 24(6), 848-855. http://doi.org/10.1200/JCO.2005.03.2235

Hodgkinson, K., Butow, P., Fuchs, A., Hunt, G.E., Stenlake, A., Hobbs, K.M., ... Wain, G. (2007). Long-term survival from gynecologic cancer: Psychosocial outcomes, supportive care needs and positive outcomes. Gynecologic Oncology, 104(2), 381-389. http://doi. org/10.1016/j.ygyno.2006.08.036

Kelly, L., Caldwell, K., \& Henshaw, L. (2006). Involving users in service planning: A focus group approach. European Journal of Oncology Nursing, 10(4), 283-293. http://doi.org/10.1016/j.ejon.2005.12.008

Lebel, S., Rosberger, Z., Edgar, L., \& Devins, G.M. (2007). Comparison of four common stressors across the breast cancer trajectory. Journal of Psychosomatic Research, 63, 225-232. http://doi. org/10.1016/j.jpsychores.2007.02.002

Lebel, S., Beattie, S., Ares, I., \& Bielajew, C. (2013). Young and worried: age and fear of recurrence among breast cancer survivors. Health Psychology, 32(6), 695-705.

Mallinger, J.B., Griggs, J.J., \& Shields, C.G. (2005). Patient-centered care and breast cancer survivors' satisfaction with information. Patient Education and Counseling, 57(3), 342-349. http://doi. org/10.1016/j.pec.2004.09.009
Mayo Clinic Cancer Education Program, (n.d.). Needs Assessment Survey, Adapted and used by the Wellness Beyond Cancer Program of The Ottawa Hospital with verbal permission from the Mayo Clinic Cancer Education Program.

Nicolaije, K.A.H., Husson, O., Ezendam, N.P.M., Vos, M.C., Kruitwagen, R.F.P.M., Lybeert, M.L.M., \& van de Poll-Franse, L.V. (2012). Endometrial cancer survivors are unsatisfied with received information about diagnosis, treatment and follow-up: A study from the population-based PROFILES registry. Patient Education and Counseling, 88(3), 427-435. http://doi.org/10.1016/j. pec.2012.05.002

Papadakos, J., Bussière-Côté, S., Abdelmutti, N., Catton, P., Friedman, A.J., Massey, C., ... Ferguson, S.E. (2012). Informational needs of gynecologic cancer survivors. Gynecologic Oncology, 124(3), 452457. http://doi.org/10.1016/j.ygyno.2011.10.030

Risendal, B., Dwyer, A., Seidel, R., Lorig, K., Katzenmeyer, C., Coombs, L., ... Ory, M. (2014). Adaptation of the Chronic Disease Self-Management Program for Cancer Survivors: Feasibility, acceptability, and lessons for implementation. Journal of Cancer Education, 29(4), 762-771. http://doi.org/10.1007/s13187-014-0652-8

Rosenbaum, E., Gautier, H., Fobair, P., Neri, E., Festa, B., Hawn, M., ... Spiegel, D. (2004). Cancer supportive care, improving the quality of life for cancer patients. A program evaluation report. Supportive Care in Cancer, 12(5), 293-301. http://doi.org/10.1007/ s00520-004-0599-0

Rushton, M., Morash, R., Larocque, G., Liska, C., Stoica, S., Degrasse, C., Segal, R. (2015). Wellness Beyond Cancer Program: Building an effective survivorship program. Current Oncology, 22(6), 419434. http://dx.doi.org/10.3747/co.22.2786

Rutten, L.J.F., Arora, N.K., Bakos, A.D., Aziz, N., \& Rowland, J. (2005). Information needs and sources of information among cancer patients: a systematic review of research (1980-2003). Patient education and counseling, 57(3), 250-261.

Stalker, M.Z., Johnson, P.S., \& Cimma, C. (1990). Supportive activities requested by survivors of cancer. Journal of Psychosocial Oncology, 7(4), 21-31. http://doi.org/10.1300/J077v07n04_02

Steinhagen-Thiessen, E., \& Borchelt, M. (1999). Morbidity, medication, and functional limitations in very old age. The Berlin aging study: Aging from 70 to 100, 131-166.

Tabachnick, B.G., Fidell, L.S., \& Osterlind, S.J. (2001). Using multivariate statistics.

Tariman, J.D., Doorenbos, A., Schepp, K.G., Singhal, S., \& Berry, D.L. (2014). Information needs priorities in patients diagnosed with cancer: A systematic review. Journal of the Advanced Practitioner in Oncology, 5(2), 115-122.

Zebrack, B. (2009). Information and service needs for young adult cancer survivors. Supportive Care in Cancer, 17(4), 349-357. http:// doi.org/10.1007/s00520-008-0469-2 